\title{
The System of Outdoor Visible Light Communication with Fast Blind Algorithm
}

\author{
Zheng-Guo Sun ${ }^{1}$, Yi-Jun Zhu ${ }^{1} \&$ Yan-Yu Zhang ${ }^{1}$ \\ ${ }^{1}$ Department of Communication Engineering, Information Science and Technology Institute, Henan \\ Province, 450001, China
}

\begin{abstract}
Keywords: Outdoor VLC (OVLC), blind channel estimation, block transmission, IM/DD, generalized likelihood ratio test (GLRT).
\end{abstract}

\begin{abstract}
In this paper, we study a new fast blind algorithm based on the generalized likelihood ratio test (GLRT) principle. It is used in an outdoor visible light communication (OVLC) system that uses OOK modulation with intensity modulation and direct detection (IM/DD) over atmospheric turbulence-induced slow fading channel, and we prove that the fast blind algorithm can detect the optical signal outdoor effectively by experiments
\end{abstract}

\section{Introduction}

As a new communication technology, VLC has been paid a lot of attention [1]-[6]. It is a data communications medium using visible light between 400 and $800 \mathrm{THz}(780-375 \mathrm{~nm})$. The intensity modulation and direct detection (IM/DD) method is practically utilized in VLC due to its simple implementation of a low-cost receiver. It can achieve the broadband transmission with unlicensed spectrum, alleviate shortage of wireless spectrum resources [7], there are no health regulations to restrict the transmit power. VLC is more and more widely used in human life, but the most technologies in VLC are used indoor.

VLC develops rapidly under the influence of light-emitting diode (LED) development. New generation of high-intensity light emitting diodes (LEDs) which has permitted the replacement of incandescent-based lights, unfolds the potential of implementing VLC system on outdoor environment [8]. High-intensity LEDs meet the demand of square illumination, streetlight, traffic light

and other outdoor scenes because of its high light and energy saving. Based on illumination equipment existing, it can achieve high speed communication with visible light. It gives an additional supplement to traditional communication with releasing the communication pressure and providing a better performance.

The channel in the indoor VLC is static and stable [9], but outdoor is different. The optical channel outdoor is in the complex atmospheric situation. The inhomogeneous of temperature and pressure lead to the atmospheric turbulence. The atmospheric turbulence makes the refractive index variable along the transmission path, optical signal attenuation degrades communication performance. Than the signal transmission rate, the channel change is more slow [10]-[13]. The outdoor optical channel is time-variable slow fading.

The paper is organized as follows. In Section 2, A new optical signal detection algorithm is proposed. In Section 3 we test our algorithm in the outdoor VLC system. Finally, we conclude our paper in section 4 .

\section{THE DETECTION METHODS OF OUTDOOR VLC}

In this section, we will put forwards our new algorithm and compare it with mean value algorithm, first let me review the mean value algorithm next.

\subsection{Mean Value Detection}

This method is common used in practice, as the probability density function (PDF) of channel is 
unknown. The channel estimation used mean value detection $\bar{h}$ is obtained by

$$
\bar{h}=\frac{1}{L} \sum_{i=1}^{L} r_{i}
$$

where $L$ is the block length, ${ }^{r}$ is the signal received. Then we get signal transmitted ${ }^{S_{i}}$ by the decision criterion

$$
s_{i}=\left\{\begin{array}{l}
1, r_{i}>\frac{\bar{h}}{2}, \\
0, r_{i} \leq \frac{\bar{h}}{2}
\end{array}\right.
$$

\subsection{Fast Blind Detection}

The channel gain $h$ and noise $n_{i}$ in $n$ are independent distribution. The pdf of received signal $r$ conditioned on $s$ and $h$ is

$$
p(\mathbf{r} \mid \mathbf{s}, h)=\frac{1}{\left(\sqrt{2 \pi \sigma_{n}^{2}}\right)^{L}} \exp \left(-\frac{\|\mathbf{r}-h s\|^{2}}{2 \sigma_{n}^{2}}\right)
$$

(3) can be simplified into (4). The Euclidian distance describes the error between received signal vector $\mathbf{r}$ and emitted signal vector $\mathbf{S}$

$$
\|r-h s\|^{2}=\sum_{i=1}^{L} r_{i}^{2}-2 h \sum_{i=1}^{L} r_{i} s_{i}+h^{2} \sum_{i=1}^{L} s_{i}^{2}
$$

(4) is a non-deterministic polynomial hard (NP-hard) problem. The common method is Viterbi algorithm [14] to search $\mathbf{s}$ with high complexity. To make the NP-hard problem be linear, we define the signal sum function.

$$
S=\sum_{i=1}^{L} s_{i}^{2}, 0 \leq S \leq L
$$

As the signal is non-negative in VLC, the ${ }^{S_{i}}$ only can be 0 or 1 in OOK modulation. When $S$ is fixed, we get the total number of symbol 1 , this idea is very important to simplify algorithm. To make (4) minimum, we order the received block signal vector $\mathbf{r}$ and summate the first $\mathrm{S}$ terms.

$$
R_{\mathrm{S}}=r_{(1)}+r_{(2)}+\ldots+r_{(\mathrm{S})},\left(r_{(1)} \geq r_{(2)} \ldots \geq r_{(\mathrm{S})}, 1 \leq \mathrm{S} \leq \mathrm{L}\right)
$$

Where $r_{(i)}$ is the order static of received signal. When $S$ is fixed, $R_{S}$ is a fixed number. Substituting (5) and (6) into (4) results in

$$
C_{S}=\sum_{i=1}^{L} r_{i}^{2}-2 h R_{S}+h^{2} S
$$

When $S=0, R_{S}=0, C_{0}=\sum_{i=1}^{L} r_{i}^{2}$ is
\[ \tilde{h}=\frac{R_{S}}{S} \]

Substitute (8) into (7) results in

$$
C=\sum_{i=1}^{L} y_{i}^{2}-\frac{R_{S}^{2}}{S}
$$

Finally we obtain the decision criterion 


$$
\max _{1<S \leq L} \frac{R_{S}^{2}}{S}
$$

By searching $S$ exhaustively to fit (11), we can achieve the channel estimate and the signal demodulation jointly

\section{EXPERIMENT}

We compare the performance of the fast blind detection algorithm and mean value algorithm with OOK signal and IM/DD. The board with Altera Cyclone II is used as a transmitter, and Kintex-7 FPGA KC705 Evaluation Kit with peripheral AD chip is used as a receiver. The known data is stored in transmitter and receiver. The transmitter transmits data repeatedly, the receiver compute the BER after receiving the signal. Two kinds of detection algorithm fast blind detection and mean value are used in receiver.

The processing of signal is shown in Fig.1. The block code is used to eliminate error floor, the data which passes through an block encoder transmits in the channel, the receiver gets signal, the detection algorithm is used to detection signal, then the signal will be decoded by block decoder. The system is shown in Fig.1, The different distance $\mathrm{D}=1 \mathrm{~m}, 10 \mathrm{~m}$ and $100 \mathrm{~m}$ is experimented.

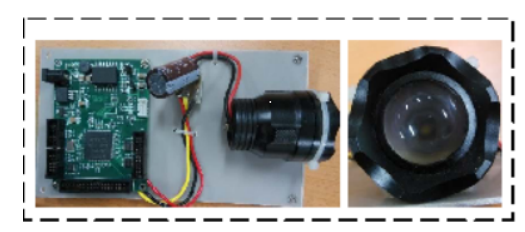

TransmitterL

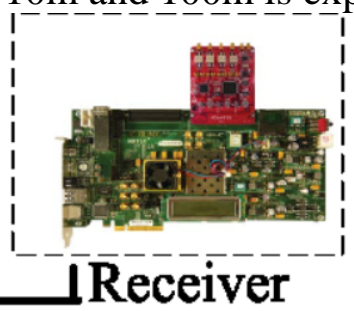

(a)

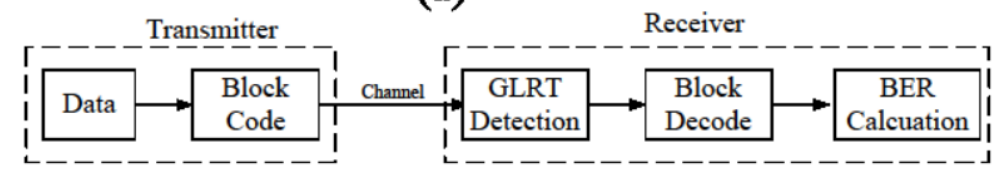

(b)

Fig. 1. (a) The experimental setup. (b) The signal processing flow between transmitter and receiver.

The results are shown in Fig.2, Fig.3 and Fig.4. As the block code is used, there no exits error floor. The fast blind detection algorithm outperform mean value algorithm in all three cases. When distance D increases, the gap increases in Fig.2, Fig.3 and Fig.4 because of atmospheric turbulence.

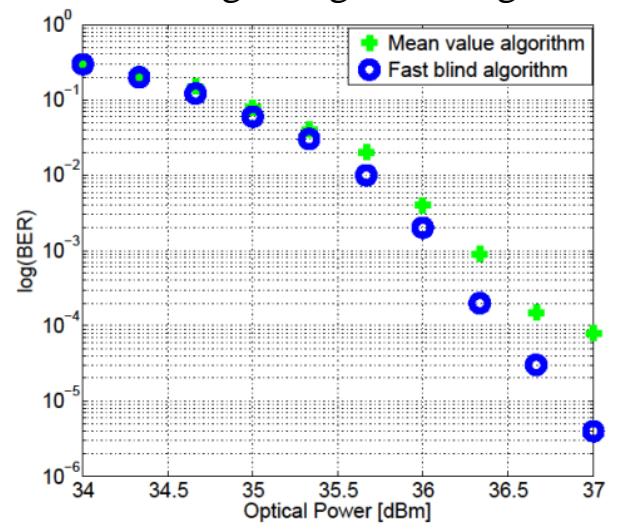

Fig. 3. The comparison between fast blind algorithm and mean value with distance $D=10 \mathrm{~m}$ 


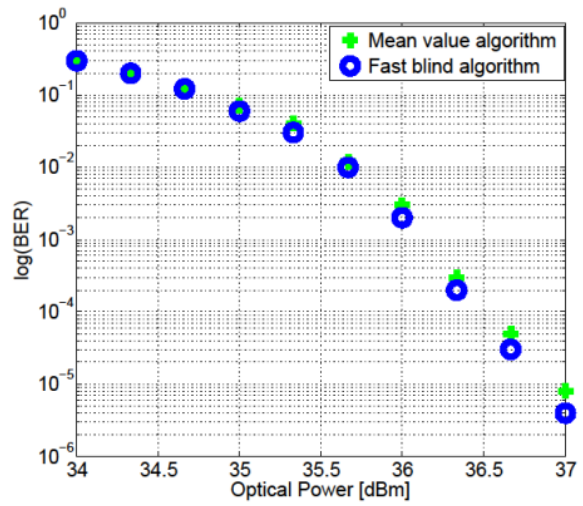

Fig. 2. The comparison between fast blind algorithm and mean value with distance $D=1 \mathrm{~m}$

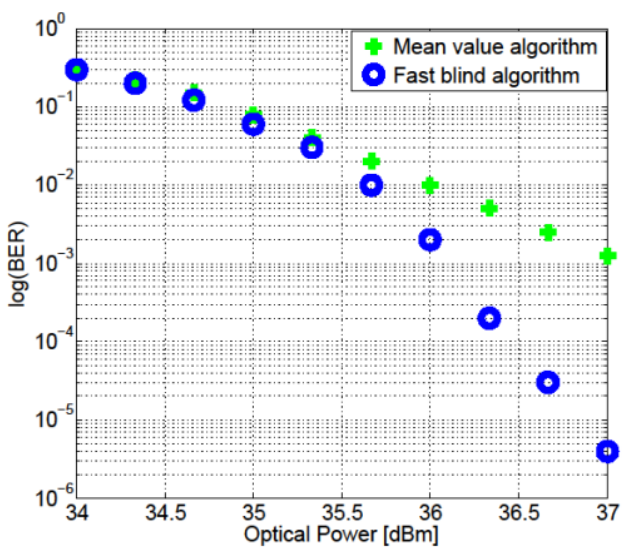

Fig. 4. The comparison between fast blind algorithm and mean value with distance $D=100 \mathrm{~m}$

\section{CONCLUSION}

In summary, a fast blind detection algorithm based on GLRT is proposed in outdoor VLC, the algorithm is tested by experiments with different block length. The algorithm are significant for slow fading channel in the outdoor VLC, they can improve system performance extremely, block code as a new method to eliminate error floor is effective.

\section{Acknowledgements}

This work was supported in part by NNSF of China (No. 61271253) and NHTRDP (863 Program) of China (Grant No.2013AA013603), in part by Grant

No. KFKT-2012103 of Beijing University of Posts and Telecommunications

(BUPT)

\section{References}

[1] D. O. A.M.Street, P.N. Stavrinou and D. Edwards, "Indoor optical wireless systems-a review," Opt. Quantum Electron., Mar. 1997.

[2] A. Acampora, “Last mile by laser,” Sci. Amer., Jul. 2002.

[3] R. S. L. K.-D. a. W. J. Grubor, J., "Broadband Information Broadcasting Using LED-Based Interior Lighting,” J. Lightw. Technol., Dec.

2008.

[4] M. Z. Afgani, H. Haas, H. Elgala, and D. Knipp, "Visible light communication using ofdm,” in Testbeds and Research Infrastructures for the Development of Networks and Communities, 2006. TRIDENTCOM 2006. 2nd International Conference on, pp. 6-pp, IEEE, 2006. 
[5] H. Sugiyama, S. Haruyama, and M. Nakagawa, "Brightness control methods for illumination and visible-light communication systems,"

in Wireless and Mobile Communications, 2007. ICWMC'07. Third International Conference on, pp. 78-78, IEEE, 2007.

[6] S. Rajagopal, R. D. Roberts, and S.-K. Lim, "Ieee 802.15. 7 visible light communication: modulation schemes and dimming support,"

IEEE Commun. Mag., vol. 50, no. 3, pp. 72-82, 2012.

[7] S. C. J. L. S. Randel, F. Breyer and J. W. Walewski, “Advanced modulation schemes for shortrange optical communications [invitedpaper],"

IEEE J. Sel. Top. Quantum Electron., vol. 16, no. 5, pp. 1280-1289, 2010.

[8] I. Lee, M. Sim, and F. Kung, "Performance enhancement of outdoor visible-light communication system using selective combining receiver,"

IET Optoelectronics, vol. 3, no. 1, pp. 30-39, 2009.

[9] K. Lee, H. Park, and J. R. Barry, "Indoor channel characteristics for visible light communications,” IEEE Commun. Lett., vol. 15, no. 2,

pp. 217-219, 2011.

[10] R. M. Gagliardi and S. Karp, “Optical communications,” New York, Wiley-Interscience, 1976. 445 p., vol. 1, 1976.

[11] N. S. Kopeika, A. Zilberman, and Y. Sorani, "Measured profiles of aerosols and turbulence for elevations of 2 to $20 \mathrm{~km}$ and consequences

of widening of laser beams,” in Photonics West 2001-LASE, pp. 43-51, International Society for Optics and Photonics, 2001.

[12] A. Zilberman, N. S. Kopeika, and Y. Sorani, "Laser beam widening as a function of elevation in the atmosphere for horizontal propagation,"

in Aerospace/Defense Sensing, Simulation, and Controls, pp. 177-188, International Society for Optics and Photonics, 2001.

[13] T. J. Schulz, “Optimal beams for propagation through random media,” Opt. Lett., vol. 30, no. 10, pp. 1093-1095, 2005.

[14] T. Song and P.-Y. Kam, "A robust glrt receiver with implicit channel estimation and automatic threshold adjustment for the free space optical channel with im/dd,” J. Lightw. Technol., vol. 32, no. 3, pp. 369-383, 2014. 